\title{
Children with Special Needs: Comparing Tactile and Tangible Interaction
}

\author{
César Ortea Suárez, Javier Marco, Sandra Baldassarri, and Eva Cerezo \\ Computer Science Department, \\ Instituto de Investigación en Ingeniería de Aragón, \\ Universidad de Zaragoza \\ \{622384,javi.marco, sandra, ecerezo\} @unizar.es
}

\begin{abstract}
In this paper a comparison of the same computer game with two interaction styles is achieved: through tactile interaction in a digital board or using tangible interaction on a tabletop. Tests were carried out with children with special needs, who have different degrees of disability. The aim of the paper is to compare usability and accessibility of each interface, as well as the experiences of children playing with them. Preliminary results indicate the necessity to provide feedback continuously, both hearing and visual, in order to facilitate the understanding of each task and its progression. In addition, the outcome obtained from a questionnaire show a significant preference for the tangible version of the game.
\end{abstract}

Keywords: Tactile, tangible, digital board, tabletop, special education, game.

\section{Introduction}

Computer games are very valuable education-learning tools for children, because they allow users to learn through active participation, to promote problem solving and to emphasize exploration and self-discovery. Also, games are an entertainment for children without perceiving that, at the same time, they are learning.

In recent years, children have gained role as users of interactive technologies. Consequently, there has been a growing interest in adapting and validating different evaluation methods to the cognitive and social development of children [1]. Also, depending on the children's disability, it is necessary to adapt the system's interaction style to their capabilities. Graphical User interfaces (GUIs) based on mouse and keyboard interaction are the most used today, although more natural and physical interaction models are becoming increasingly popular [2]. Natural interaction methods, including tactile interaction and tangible user interfaces (TUIs), have been explored as a way of bringing children to the digital contents. In particular, TUIs combine physical and virtual objects in interactive and physical environments [3] and, therefore, they are more exploratory, collaborative and expressive [4].

In this paper, we carry out a comparison of the same game with two kinds of natural interaction: tactile interaction in digital board and tangible interaction in horizontal tabletop. Children who participate in the evaluation tests belong to special education school in order to learn how to fit these two interaction styles to the needs 
of children with cognitive problems [5] [6] [7]. Consequently, the evaluation methods have to be chosen and adapted according to their capacities, with the purpose of obtaining information about usability, accessibility and user experience (UX) of both interfaces.

\section{The Farm Game with Different Interaction Styles}

We compare the same video game for children in two different devices: horizontal tabletop based on tangible interaction (Fig. 1 left) and a digital board based on tactile interaction (Fig. 1 right), as both enable small groups of children playing together.

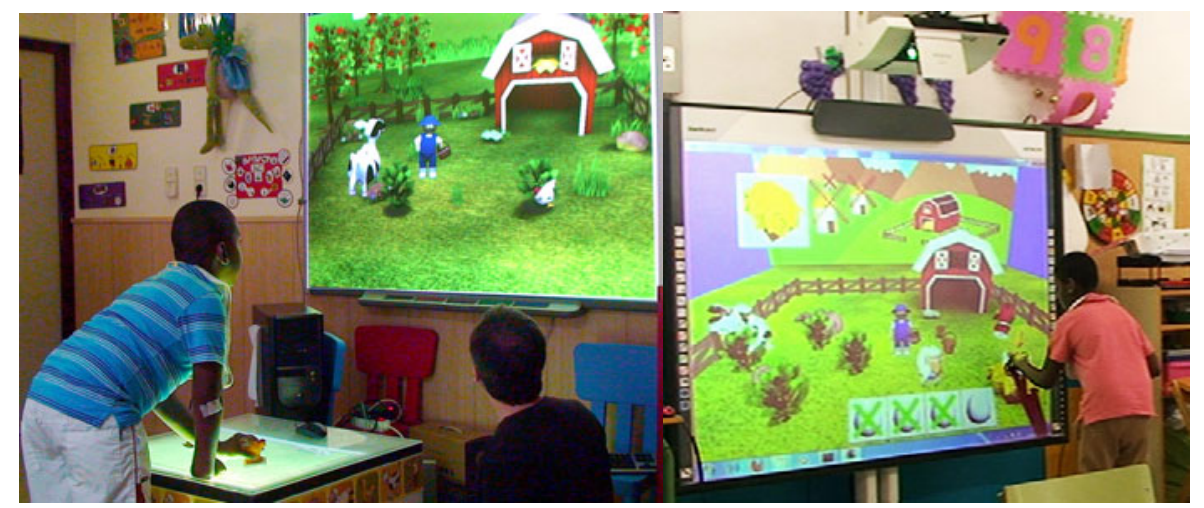

Fig. 1. Tangible (left) and tactile (right) interfaces

The tangible interface is composed of a horizontal tabletop (NIKVision) specially designed for children [8]. The interaction with the games is through physical manipulation of toys on the table. The device has an active output image on the tabletop and on a monitor next to it.

On the other hand, the tactile interaction on the digital board is achieved by pressing on an image which is projected onto a vertical screen (TouchIT model). Also, the board is situated near to the ground in order to children can touch easily everywhere.

For both interactive devices, it has been designed the same game: a Farm game, which consists of a final goal, several sub-goals, characters controlled by the player, an autonomous characters and interactive objects. The ultimate goal of the game is to help the farmer to make a cake for his son's birthday. To achieve this goal, children must complete three sub-goals: get three strawberries, four eggs and a bucket of milk.

The players can control several characters: a hen, a cow, a sheep and a pig. Strawberries can be picked up with any animal, while eggs and milk can only be obtained with the hen and cow, respectively. The farmer is an autonomous character, which gives hearing feedback (instructions about who, where and how pick up the ingredients). In addition, there is different visual feedback in both versions: 
- Tabletop: The projection on the table indicates where to place the animals, where strawberries are and how many eggs or milk have been achieved.

- Digital board: At the top of the screen, a box indicates the object where the animal must be placed. At the bottom, several boxes indicate the amount of strawberries, eggs or milk already picked up and the amount that is still needed.

Finally, the interactive objects are: plants (with or without strawberries), the nest and the bucket. Every animal can be moved or placed on these virtual objects and it can perform an action on them, giving back feedback about task's progress (farmer's instructions).

The sequence of actions to perform in order to complete a task varies in each interaction style:

- Tangible: first, a toy animal is placed on a virtual object on the table surface and then, the toy can be shaken in the plants to get a strawberry, or jumps can be done with the hen and cow toys to lay an egg in the nest or to give milk in the bucket respectively.

- Tactile: it requires a sequence of touches on the board to complete an action: first, an animal is touched to activate it, then an object is touched and the animal moves to it and, finally, the object is touched again to trigger an action.

\section{Comparative Test}

An evaluation session has been carried out involving special education children who have different degrees of cognitive and/or physical disabilities. In particular, eight children with ages between 6 and 11 years, participate in couples.

As has been said before, the evaluation methods used are intended to get usability, accessibility and UX problems in both interaction styles. Specifically, the evaluation methods selected for these test sessions, are the following: usability test (evaluators write observations relating to children's interaction with both interfaces), video analysis, LOG (automatic record of events and actions that happen in the game), likeability questionnaire (to obtain the preference of children) and SEEM questionnaire (Structured Expert Evaluation Method) in order to capture experts' view of the interaction problems of the children.

We present the preliminary outcomes and results from our observations and questionnaires, but a more thorough analysis from the videos and LOGs must be still carried out.

The observation reveals that children interact following the instructions in the same order they receive them (data obtained from LOGs).

The results of the questionnaires mainly address to the need to provide more feedback, both visual and hearing. In particular, in the tangible version of the game, it is necessary to provide back visual or hearing feedback to show children how many objects are still needed. Also the major goal of the game (to make a cake) must be clearly represented so that children can understand the aim. The tactile version of the game, on the other hand, has to provide more visual feedback, especially on those 
positions where children have to trigger an action. In addition, farmer's instructions must be given continuously and must be as simple as possible.

A widespread problem is the difficulty for children to complete the task of picking up strawberries, because they don't perceive clearly where the strawberries are placed. Therefore, it is necessary to give back visual feedback in these plants.

Finally, regarding to the answers given in the likeability questionnaire, 5 out of 8 children prefer tangible version and only one person prefer the tactile version (2 children didn't say anything).

In summary, this study shows us the differences between both versions and the importance of giving back visual and hearing feedback so that children can understand the game's progress. In tactile version, hearing feedback must be simplified and visual feedback must be displayed in the interactive object (not in other place), while in tangible version feedback should be given to show how many actions are in the task. Regarding to the answers of the likeability questionnaire, 5 out of 8 children prefer tangible version and only one person prefer tactile version ( 2 children didn't say anything).

Acknowledgements. This work has been partially financed by the University of Zaragoza, through the Project called "AVIM-Agentes Virtuales Inteligentes y Multimodales" and the Government of Aragon through Cooperation projects between University Departments and Secondary Schools. The authors thank the collaboration of CPEE Alborada School.

\section{References}

1. Baauw, E., Markopoulos, P.: A comparison of think-aloud and post-task interview for usability testing with children. In: Proceedings of the 2004 Conference on Interaction Design and Children: Building a Community, IDC 2004, pp. 115-116 (2004)

2. $\mathrm{Xu}, \mathrm{D} .:$ Design and Evaluation of Tangible Interfaces for Primary School Children. In: Proceedings of the 6th International Conference on Interaction Design and Children, IDC 2007, pp. 209-212 (2007)

3. Fails, J.A., Druin, A., Guha, M.L., Chipman, G., Simms, S., Churaman, W.: Child's Play: comparison of Desktop and Physical Interactive Environments. In: Proceedings of Interaction Design and Children (IDC 2005), pp. 48-55 (2005)

4. O'Malley, C., Fraser, D.S.: Literature Review in Learning with Tangible Technologies. NESTA Futurelab, 12 (2004)

5. Li, Y., Fontijn, W., Markopoulos, P.: Tangible tabletop game supporting therapy of children with Cerebral Palsy, pp. 182-193. Springer, Heidelberg (2008)

6. Piper, A.M., O'Brien, E., Morris, M.R., Winograd, T.: SIDES: a cooperative tabletop computer game for social skills development. In: Proceedings of the 2006 20th Anniversary Conference on Computer Supported Cooperative Work (CSCW 2006), pp. 1-10. ACM, New York (2006)

7. Baldassarri, S., Cerezo, E., Blasco, G.: Juegos educativos configurables para Educación Especial. In: En: XI Congreso Internacional de Interacción Persona Ordenador, pp. 99-108 (2010) (in Spanish)

8. Marco, J., Baldassarri, S., Cerezo, E.: Bridging the Gap between Children and Tabletop Designers. In: Proceedings of the 9th International Conference on Interaction Design and Children, IDC 2010, pp. 98-107 (2010) 